\title{
On Innovation, Quality of Life and Technology of BodyNets
}

\author{
Sungmee Park \\ Georgia Institute of Technology \\ School of Polymer, Textile \& Fiber Engineering \\ Atlanta, Georgia 30332-0295, USA \\ sungmee.park@gatech.edu
}

\author{
Sundaresan Jayaraman* \\ Georgia Institute of Technology \\ School of Polymer, Textile \& Fiber Engineering \& \\ College of Management \\ Atlanta, Georgia 30332-0295, USA \\ sundaresan.jayaraman@gatech.edu \\ * To whom correspondence should be addressed
}

\begin{abstract}
Technology innovation is the key to enhancing the quality of life for everyone in the continuum of life from newborns to senior citizens. Management innovation is the fuel that sustains the technology innovation engine. Thus, together, management innovation and technology innovation have the potential to rapidly transform information processing, especially in healthcare, by successfully addressing the key challenges of access, quality and cost of care. In this paper, we examine the healthcare industry and attempt to diagnose its major challenges; we then define the healthcare continuum and explore the roles of human as an information node and BodyNets as the enabling technology to bring about a transformation of the industry to nurture it back to health. Following this, we present one approach to realizing a BodyNet using the Wearable Motherboard paradigm. We then discuss the impact of BodyNets in bringing about transformational change in the healthcare enterprise. We conclude with a brief discussion of the importance of transitioning the technology to the marketplace and highlight the broader applications of BodyNets to entertainment and lifestyle enhancement.
\end{abstract}

Healthcare Continuum, BodyNets, Wearable Motherboard, Smart Shirt, Cost of Care, Quality of Care, Chronic Diseases, Sensors

\section{INTRODUCTION}

A careful examination of today's healthcare enterprise reveals the following symptoms:

- As a share of gross domestic product (GDP), health spending in the United States is projected to reach 19.6 percent by 2016, up from its 2006 level of 16 percent; during the same period, total spending is expected to increase from $\$ 2.1$ Trillion to $\$ 4.1$ Trillion [1].

- Experts estimate that as many as 98,000 people die in any given year from medical errors that occur in hospitals. Add the financial cost to the human tragedy, and medical error easily rises to the top ranks of urgent, widespread public problems [2].

- Private insurers (such as Aetna and Blue Cross and Blue Shield Association) are following Medicare's lead in implementing a system in October 2008 to deny payments for treating so-called "never events" - serious and costly errors such as pressure ulcers, air embolism, mismatched blood transfusion, object left in patient after surgery, and patient falls that should have never happened in the first place [3].

- The US population is rapidly aging with the number of people 65 and older expected to grow from 35 million in 2000 to 72 million in 2030 and make up for nearly 20 percent of the total U.S. population at that time. Heart disease, cancer, and cerebrovascular diseases (stroke) continue to be the leading causes of death among older Americans. Of the 1.8 million deaths in 2000 of people aged 65 and over, 33 percent were caused by heart disease, 22 percent were caused by cancer, and 8 percent were caused by cerebrovascular diseases [4].

- In the first half of $2005,14.4 \%$ of the population was uninsured, and $5.7 \%$ of the population was unable to obtain needed medical care due to cost at some time during the previous 12 months [5].

- Analysis of data from the Medical Expenditure Panel Survey (MEPS) shows that rising out-of-pocket expenses and stagnant incomes increased health spending's financial burden for families in 2001-2004, especially for the privately insured. It has reached a point where private insurance is no longer able to provide financial protection for an increasing number of families [6].

- Between 1997 and 2004, the median emergency room wait time increased 36 percent; specifically, the median wait time for heart-attack patients increased from eight minutes in 1997 to 20 minutes in 2004 - a 150\% increase; during the period 1994-2004, the number of emergency visits increased from 93.4 million to 110.2 million 
annually, while the number of emergency departments fell by as much as 12.4 percent [7].

- A new healthcare delivery channel has emerged in the form of retail or in-store clinics staffed by nursepractitioners, which have grown from 62 in January 2006 to over 700 by the end of 2007 , and projected to rapidly increase to 1,500 by the end of 2008 [8].

- The increased regulatory authority granted by the "Food and Drug Administration Amendments Act of 2007" to the Food and Drug Administration (FDA) to monitor the safety of marketed drug products and medical devices places additional responsibilities on pharmaceutical companies, device manufacturers and healthcare providers [9].

In addition, the Institute of Medicine, in a landmark study, concluded, "The U.S. healthcare delivery system does not provide consistent, high-quality medical care to all people" [10]. These challenges, however, are not confined to the United States alone. According to a study by PricewaterhouseCoopers, "Globally, healthcare is threatened by a confluence of powerful trends - increasing demand, rising costs, uneven quality, misaligned incentives. If ignored, they will overwhelm health systems, creating massive financial burdens for individual countries and devastating health problems for the individuals who live in them" [11].

The Diagnosis: Together, these symptoms lead to the diagnosis that the healthcare industry is itself seriously ailing and its prognosis depends on successfully addressing the following critical symptoms:

- Reduce healthcare costs while maintaining a high quality of care;

- Provide access to care for as many people as possible;

- Control length of hospital stay and decentralize the provision of healthcare;

- Address the growth in the aging population and caring for chronically ill patients;

- Shift the focus of healthcare expenditures from treatment to prevention through wellness programs;

- Provide easy access to specialized professionals anywhere and anytime;

- Collaborate with and co-opt emerging channels of healthcare delivery; and

- Comply with the new regulations for drug and device safety.

In short, the healthcare enterprise is confronted with the challenging task of containing the cost of care and enhancing the quality of care while simultaneously broadening access to care. And, more importantly, a failure to do so will make the healthcare system unsustainable due to the combination of growing demands (with increasing population), escalating prices and decreasing resources (shortage of doctors and nurses).
The Prognosis: On the bright side, there are a few technology trends, that properly harnessed, can improve the prognosis; these include:

- The significant advancements in mobile information and communications technologies - both hardware and software - are fundamentally transforming information processing. No longer confined to a static computing infrastructure anyone can process information anytime and anywhere, giving birth to the paradigm of pervasive information processing or "information processing on the go."

- The advancements in, and convergence of, microelectronics, materials, optics and bio technologies, coupled with miniaturization, have led to the development of small, cost-effective intelligent sensors for a wide variety of applications.

- The transparency of the user interface coupled with the invisibility of the "embedded" technology in the various devices and systems have contributed to the proliferation of sensors and sensor networks in various settings - from homes to workplaces.

Technology is the catalyst that can enable affordable healthcare to anyone, anywhere at anytime. Innovation involves creating something that is new - a product or service that has not existed heretofore. As shown in Figure 1,

\begin{tabular}{|c|}
\hline Inspiration \\
Necessity \\
Neat \\
Original \\
Valuable \\
Applicable \\
Timely \\
Intelligence \\
Outstanding \\
Novel \\
\hline
\end{tabular}

Figure 1. Defining Innovation innovation is driven by inspiration and is triggered out of a necessity. For instance, the first generation of smart phones that integrated mobile phone features with a personal digital assistant (PDA) grew out of the necessity to meet the requirements of individuals struggling simultaneously with two different devices (which were large in size by today's standards). When the first smart phone came out, it was indeed considered to be neat and original because such a device didn't exist; that innovation was extremely valuable and applicable to the users (mostly business professionals at that time); timeliness of an innovation is important and often involves intelligence; novelty is another characteristic of innovation as shown in the figure. And, over a period of time, an innovation becomes part of the mainstream; for instance, even though Apple's iPhone ${ }^{\circledR}$ integrates a PDA with a mobile phone, that feature is no longer considered to be "innovative"; rather, its touch screen interface is considered to be an innovation.

Quality of life is typically associated with the overall wellbeing of individuals and is a combination of subjective and objective metrics. According to the CDC, in public health and 
in medicine, the concept of health-related quality of life (HrQOL) refers to a person or group's perceived physical and mental health over time [12]. A detailed study in this area has shown that Americans with chronic diseases, such as diabetes, arthritis and cardiovascular, reported high levels of unhealthy days, which affected their Hr-QOL. Older adults suffered the most poor physical health and activity limitation thereby affecting their Hr-QOL. Likewise, a wealthy young mother with a newborn suffering from sleep apnea may feel that her quality of life is poor since she can't get a good night's sleep and is constantly living in fear that her baby may stop breathing anytime; although economically well-off, the health condition of her child lowers her assessment of her quality of life.

The Prescription and Treatment: Technology innovation is the key to enhancing the quality of life for everyone in the continuum of life from newborns to senior citizens - whether it is preventing deaths due to sudden infant death syndrome (SIDS) in infants 1 to 12 months of age or treating chronic diseases such as diabetes in the aging population. And, management innovation is the fuel that sustains the technology innovation engine. Thus, together, management innovation and technology innovation have the potential to rapidly transform healthcare by successfully treating the triad of symptoms afflicting the industry, viz., inadequate access, poor quality and rising cost of care. This powerful innovation combination can nurse the ailing healthcare enterprise back to health and create a system defined by the Institute of Medicine as one that "is safe, effective, patient-centered, and provides timely, efficient, and equitable care that does not vary in quality across the nation" [13].

The remainder of the paper is organized as follows: In Section 2, we define the healthcare continuum and examine the roles of human as an information node and BodyNets as the enabling technology to bring about the necessary transformations to realize the desired healthcare system. In Section 3, we discuss one example of the realization of the BodyNet technology in the context of a pervasive healthcare system. In Section in 4, we explore the impact of BodyNet in bringing about transformational change in the healthcare enterprise. Finally, in Section in 5, we conclude with a brief discussion of the importance of transitioning the technology to the marketplace and cover the broader applications of BodyNets to entertainment and lifestyle enhancement.

\section{THE PATIENT-CENTRIC HEALTHCARE CONTINUUM}

Figure 2 shows one view of the healthcare continuum in which the patient, the principal stakeholder, is at the center of the system. In this patient-centric view of the continuum, the other major stakeholders affecting the patient include the healthcare providers, the pharmacies, and suppliers that are in the frontline of patient care. The patient's employer and insurance company determine the level and cost of care received. The biotechnology companies are responsible for innovative drugs which are initially funded by venture capitalists and subsequently developed through clinical trials and brought to market for the patient by pharmaceutical companies shown in the figure. In addition, the patient's quality and cost of care are influenced by the research and teaching institutions that are developing the required human resources and technological innovations. The last major stakeholder is the government, which strives to ensure the safety and privacy of the patient and the proper functioning of the continuum. The effects of these interactions on the patient, and in fact, between the other stakeholders themselves, will significantly influence the healthcare delivered to the patient. For instance, the healthcare provider's reimbursement is often dictated by the insurance company and the hospital's cost structure is influenced by the effectiveness of its supply chain. This integrated view of the healthcare continuum is critical for creating a patient-centric healthcare system that delivers the right care to the right patient in the right place, at the right time and at the right cost.

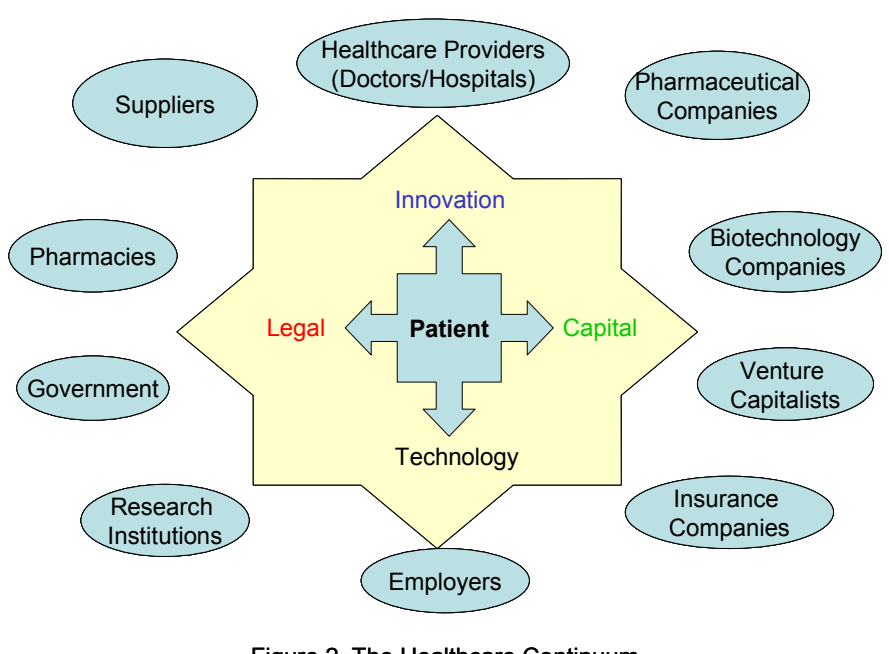

Figure 2. The Healthcare Continuum

The Enablers in the Continuum: The key enablers that drive this continuum - innovation, capital (finance), technology (medical/information) and law (legal/regulatory) - are also shown in the figure. Innovation is the lifeblood of the continuum, whether they are technological innovations in pharmaceuticals and medical devices or management innovations in pay-for-performance systems and patient care. Capital is critical for the smooth flow of operations in the continuum that is made safe by the other enabler, laws. Information technology - in the form of electronic medical records (EMR), computerized physician order entry systems (CPOE) and wireless communications technologies - can streamline the information flow and enhance the care of the patient while minimizing the cost of care. Latest advancements in medical technologies and procedures can speed up recovery and significantly curtail the duration of 
hospital stay, which in turn can reduce the possibility of never events, such as bedsores and other infections.

The Human as the Information Node: At the heart of the continuum in Figure 2 is information and the human is the central information node providing real-time information to the healthcare providers and other necessary stakeholders to create a patient-centric healthcare system. This "information" must be transformed into "knowledge" for diagnosis and treatment of the individual. In fact, having the right information at the right time in the right place is critical for delivering the right care in the continuum, in which each stakeholder can be considered a node in the continuum information network. The key then is how best to acquire and harness this information in the continuum.

Let's consider an individual who is participating in a clinical study for a new drug being developed by a pharmaceutical company that is expected to reduce the risk of heart attacks for obese people. During the course of a typical day, the individual is likely to engage in a wide range of activities (e.g., working, shopping, exercising, etc.) in different geographically distributed locations such as a hospital, gymnasium, theatre, restaurant, etc. On one such day, while at work, the individual suddenly has a stroke and is rushed to the hospital. If information about the individual's condition is transmitted immediately to the hospital even as the individual is being rushed there, the hospital doctors can prepare in advance to provide speedy and safe treatment and, hopefully, ensure the survival of the patient.

BodyNet: The Enabling Technology: In this scenario, the individual - as an information node - is providing the valuable real-time data and situational awareness to the healthcare professionals in the hospital. A structured analysis of the sensing requirements for the monitoring of the individual being carried out continuously by the pharmaceutical company as part of its drug study leads to the following conclusions [14]:

1. Different types of sensors will be needed to simultaneously monitor the various vital signs (e.g., heart rate, body temperature, pulse oximetry, blood glucose level);

2. Different numbers of sensors may be needed to obtain the signals to compute a single parameter (e.g., at least three sensors are required to compute the ECG);

3. The sensors need to be positioned in different locations on the body to acquire the proper signals (e.g., sensors for ECG); and

4. Different subsets of sensors and devices may be used at different times necessitating their easy attachment and removal, or plug and play. For instance, the individual may have to record how the body feels and reacts while taking the required noon exercise walk (prior to taking the medication) and would need a microphone and recording device only at that time.
In short, what is needed is a personalized mobile information infrastructure that would be unobtrusive and would become an "integral" part of the individual so as to indeed "disappear." And, if this information infrastructure can be realized in the form of a sensor network on the individual's body - a BodyNet - that can collect, process, store and transmit information about the individual, e.g., body vital signs, to any remote location, such as the healthcare provider or a health monitoring service (e.g., for the elderly), it would enhance the quality and timeliness of care given to the individual in this scenario.

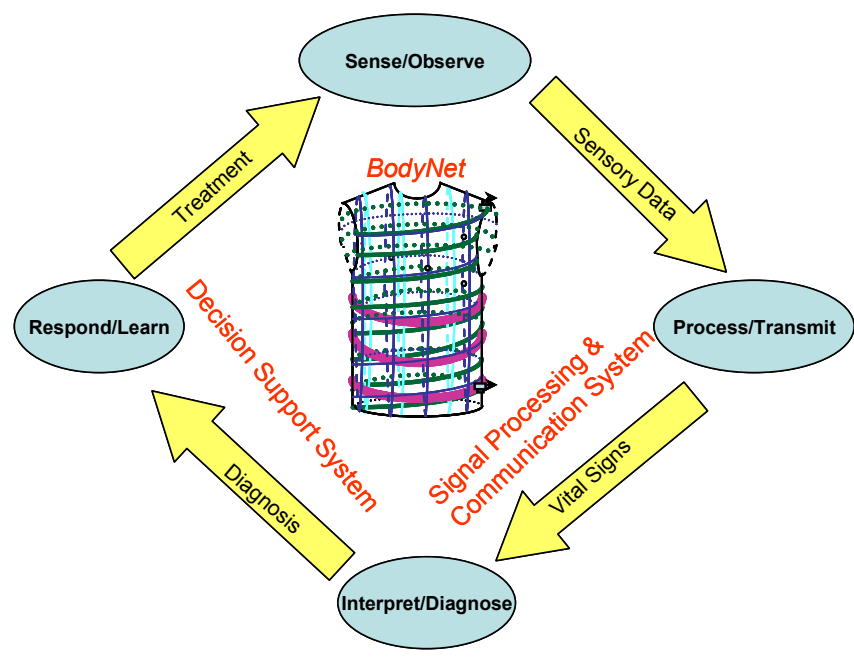

Figure 3. BodyNet in a Pervasive Healthcare Architecture

BodyNet in the Healthcare Continuum: Figure 3 shows a schematic representation of such a system incorporating a BodyNet on the individual. The major unit operations in the system, viz., sense/observe, process/transmit, interpret/diagnose and respond/learn and their respective outputs, viz., sensory data, vital signs, diagnosis and treatment are also shown in the figure. In the scenario described earlier, the sensors integrated into BodyNet on the individual capture the signals continuously. The signal processing system processes the signals - biometric, video, environmental, etc. and computes the appropriate parameters, including vital signs in terms of heart rate, body temperature, and ECG (electrocardiogram). The communications system then transmits the computed data to the hospital for analysis and storage. There, a knowledge-based decision support system that also has access to the individual's medical history (through the EMR) can help the healthcare professionals interpret the information, diagnose the individual's condition and develop an appropriate treatment to be administered in a timely manner. Simultaneously, the system can learn and update its knowledge base and transmit this information to the pharmaceutical company conducting the drug trial. The treatment can be initiated as soon as the individual arrives at the hospital (or even on the way, if appropriate) and without the loss of any time that might be critical to the survival of the individual. Thus, with the BodyNet at the heart of a pervasive 
healthcare architecture, not only can the quality of life be enhanced, but potentially a life can be saved. We will now discuss the physical realization of the BodyNet concept.

\section{REALIZING THE BODYNET: THE WEARABLE MOTHERBOARD PARADIGM}

The underlying premise behind the BodyNet concept is to have a wearable information infrastructure that can carry out the functions depicted in Figure 3. Its realization in practice has taken many forms as seen in the body of literature in the areas of smart textiles, intelligent clothing, e-textiles, wearable biomedical systems, and sensor networks, to name a few [1524]. In addition to the key functionality of sensing, the other important requirement is that it be totally customizable and be 'in-sync' with the human body. Thus, the information infrastructure - BodyNet - must also meet the following requirements:

1. Can be shaped and sized to meet the requirements of the user;

2. Can enable the rapid plug and play of sensors and devices as real-time requirements dictate;

3. Can be preconfigured with the desired suite of heterogeneous sensors and multifunction processors integrated into the substrate;

4. Is robust enough to withstand different types of operational (stress/strain) and harsh environmental (biohazards and climatic) conditions;

5. Is lightweight, portable and easy to deploy; and

6. Is easy to manufacture and has low cost.

Textiles: The BodyNet Platform: While sensor networks are relatively new in the timeline of civilization, there has been one piece of "sensing" technology that has been there since the dawn of civilization. That is textiles, which, in today's world are pervasive. Textiles (clothing) were initially used for "protection" from the environment - be it from climatic conditions or from other predators as camouflage and personal privacy. This first dimension of "protection" has been complemented by the second dimension of "aesthetics," exemplified by the success of fashion houses in modern times - from Armani to Versace. Moreover, textiles can meet the requirements listed earlier because fabrics [25]:

1. Are flexible, strong, lightweight, and shape conformable;

2. Can be made in desired dimensions of length and width, and hence area;

3. Can be produced from a variety of fibers and yarns using various manufacturing processes to accommodate different types of operational and environmental conditions;

4. Are easy to manufacture in a relatively cost-effective (inexpensive) manner compared to traditional printed circuit boards; and

5. Can easily accommodate "redundancies" in the system.
Therefore, textiles can serve as this informationprocessing infrastructure for BodyNet into which the sensors and processors can be easily integrated, thus adding the third dimension of intelligence to textiles. Just as special purpose chips and processors can be plugged into a computer motherboard to obtain the desired information processing capability (e.g., high-end graphics), the motherboard paradigm can provide an extremely versatile framework for the incorporation of sensing, monitoring, and information processing devices [15]. However, as the name implies, BodyNet - in the form of the motherboard - must be wearable by the individual. This need leads to the paradigm of a "Wearable Motherboard." We now discuss the realization of a wearable motherboard and its role in enabling a personalized and pervasive healthcare system.

Implementation of BodyNet: The Wearable Motherboard: The motivation for the Wearable Motherboard came from the need to enhance the quality of life for soldiers on the battlefield; since medical resources are limited in a combat zone, a technology that could - in real-time - determine the condition of the wounded soldier would help the medic save valuable time in attending to the soldiers who could be saved. A soldier always wore his uniform; so, it became the infrastructure for the sensors and processors and the wearable motherboard paradigm was born. Since the objective was to create a comfortable and wearable information infrastructure for the sensor network or BodyNet, factors such as Wearability, Durability, Manufacturability, Maintainability, Connectivity, and Affordability, were considered in its design and development [16]. Figure 4 shows the key functional operations associated with the Wearable Motherboard, which are analogous to those in a typical computer.
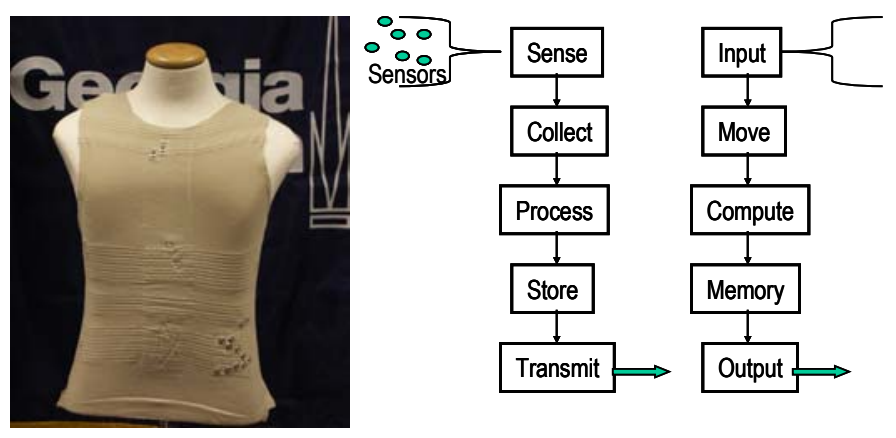

Figure 4. The Wearable Motherboard: The Desired Functionality and Computing Analogy

The comfort or base fabric - made from typical textile fibers depending on the intended application - provides the necessary physical infrastructure for the Wearable Motherboard or Smart Shirt shown in the figure. The developed interconnection technology has been used to create a flexible and wearable framework to plug in sensors for monitoring a variety of vital signs. The sensors themselves can be positioned in desired locations on the body and plugged 
into the Smart Shirt. Just as the motherboard facilitates the "plug and play" concept, other sensors can be easily integrated into the structure. For instance, a sensor to detect oxygen levels or hazardous gases can be integrated into a variation of the Smart Shirt that will be used by firefighters. Similarly, by plugging in a microphone into the Smart Shirt, voice can be recorded or an MP3 player can be plugged in along with a pair of headphones so that the user could listen to music while jogging. The signals from the sensors flow through the flexible data bus integrated into the structure to the multifunction processor/controller. This controller, in turn, processes the signals and transmits them wirelessly (using the appropriate communications protocol) to desired locations (e.g., doctor's office, hospital, battlefield triage station). The bus also serves to transmit information to the sensors (and hence, the wearer) from external sources, thus making the Smart Shirt a valuable information infrastructure. The multifunction controller provides the required power to the Wearable Motherboard. With the advent of the Smart Phone, it could serve as the hardware platform and take the place of a specialized controller. Thus the Wearable Motherboard paradigm is an effective means of creating a BodyNet, where the resulting structure has the look and feel of traditional textiles with the fabric serving as a comfortable information infrastructure. We will now examine the role of BodyNet technology in realizing the desired healthcare system.

\section{BODYNET AND THE HEALTHCARE BLUES: THE TRANSFORMATIONAL THERAPY}

The BodyNet technology, serving as a valuable information infrastructure, provides one of the therapies for nursing the healthcare industry back to health. By facilitating real-time information flow in the continuum, the information about the individual can be effectively utilized to provide the right care to the patient and address the three major symptoms of inadequate access, poor quality and rising cost of care.

The BodyNet Chain Reaction: The chain reaction triggered by the seamless integration of information from BodyNet on the patient with the corresponding EMR and CPOE systems in the hospital is illustrated in Figure 5. By providing the right clinical treatment, preventable medical errors can be reduced; through proper patient identification and tracking - resulting from the integration of "RFID" tags into BodyNet - never events such as amputating the incorrect body part or transfusing the incorrect blood type can be avoided; by integrating pressure sensors and accelerometers into the BodyNet, the patient on the hospital bed can be continuously monitored and the patient's position shifted to avoid the occurrence of bed sores (another never event); the accelerometer data can be used to detect and prevent patient falls, another never event. In all instances, the three key symptoms of cost, quality and access to care will be positively impacted as shown in the figure. This chain reaction will ripple through resulting in a larger client base because of better performance and enhanced reputation of the healthcare provider.

A similar analysis can be carried out for each of the other stakeholders in the continuum. For instance, the data from the BodyNet on an individual participating in a drug trial can be used by the pharmaceutical company to modify its drug development process; it will also enable it to comply with the mandates arising out of the new FDA regulations regarding pre-market testing of drugs. Thus, the BodyNet technology can bring about transformational change in the healthcare industry and nurture it to create a "safe, effective, patientcentered system that provides timely, efficient, and equitable care that does not vary in quality across the nation."

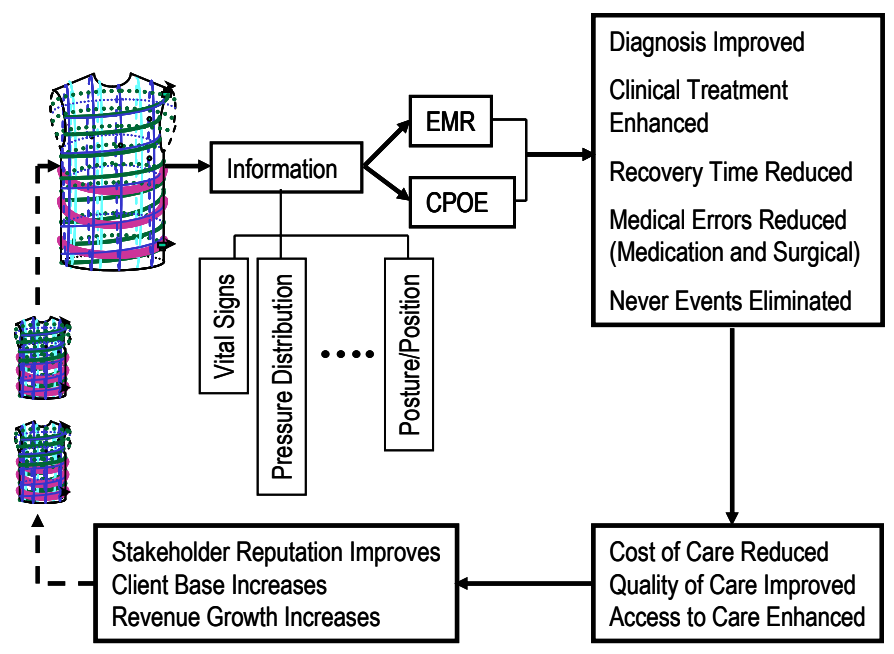

Figure 5. The Chain Reaction triggered by BodyNet in the Healthcare Continuum

\section{LOOKING AHEAD}

The true value of an innovation can be realized only when the idea enters the real-world in the form of a product or service and enhances the quality of life for the users. Therefore, it is important to transition the BodyNets technology from the laboratory to the real-world to demonstrate its potential and realize its value. Such a transition for the healthcare application will necessitate a three-step process:

- Establish the robustness of the various components of the BodyNets architecture;

- Demonstrate the clinical effectiveness of BodyNets; and

- Conduct a holistic cost-benefit analysis.

Such a roadmap analyzing the technical, business and public policy issues influencing the adoption and acceptance of the wearable motherboard technology has been proposed elsewhere [26].

Other Application Domains: While the discussion so far has primarily focused on the role and impact of BodyNets in healthcare, the applications of this technology have a much broader scope; it is applicable to all facets of personalized 
mobile information processing - from entertainment and gaming to lifestyle enhancement. BodyNets provide a platform for incorporating application-specific sensors and processors; for instance, a BodyNet architecture can be defined for a gamer so that the hands themselves will take the place of any physical controls (e.g., joystick) for all actions and gestures in the game. Moreover, the individual's physical state can be monitored in real-time using the same BodyNet infrastructure (by plugging in additional sensors) and used to control the flow of the game and its features creating a dynamic and responsive game environment. Such are the universality of the concept and the breadth of its underlying framework and infrastructure.

Concluding Remarks: Dating back to the invention of the 'wheel', technological innovations have dramatically altered the course of history and shaped human civilization. Likewise, techniques, which could be deemed management innovations, have been sustaining such technological innovations in the advancement of human kind. It is no different today: The healthcare industry is at a threshold facing a set of significant challenges. We have proposed the concept of a healthcare continuum to lay the foundation for a systems approach to addressing these challenges. We explored the role of BodyNets as an enabling technology and discussed the Wearable Motherboard paradigm as a way of realizing BodyNets. The value of BodyNet technology in curing the healthcare blues and bringing about transformational change has been demonstrated using the concept of a chain reaction, which will eventually lead to the growth and financial stability of the healthcare enterprise. Thus, innovation - technical and management - can enhance the quality of life for everyone.

\section{REFERENCES CITED}

[1] http://www.cms.hhs.gov/NationalHealthExpendData/downloads/ proj2006.pdf, Last Accessed: January 19, 2008

[2] "To Err is Human: Building a Safer Health System", Committee on Quality of Health Care in America, Institute of Medicine, Washington, DC, 2000.

[3] www.cms.hhs.gov/quarterlyproviderupdates/downloads/ cms1533fc.pdf, Last Accessed: January 19, 2008.

[4] http://www.census.gov/prod/2006pubs/p23-209.pdf, Last Accessed: January 19, 2008.

[5] http://www.cdc.gov/nchs/data/nhis/earlyrelease/ earlyrelease200509.pdf, Last Accessed: January 19, 2008.

[6] Banthin, J.S., Cunningham, P., Bernard, D.M., "Financial Burden of Health Care, 2001-2004, Health Affairs, Jan/Feb 2008, Vol. 27, No.1, pp. 188-196.

[7] Wilper, A.P., Woolhandler, S., Lasser, K.E., McCormick, D., Cutrona, S.L., Bor, D.H., and Himmelstein, U., "Waits to see an Emergency Department Physician: U.S. Trends and Predictors, 1997-2004", http://content.healthaffairs.org/cgi/content/abstract/hlthaff.27.2.w8 4, Last Accessed: January 19, 2008.

[8] http://www.chcf.org/documents/policy/HealthCareInTheExpress LaneRetailClinics2007.pdf, Last Accessed: January 19, 2008.

[9] Food and Drug Administration Amendments Act of 2007, P.L. 110-85, September 27, 2007, http://frwebgate.access.gpo.gov/cgibin/getdoc.cgi? dbname $=110$ cong public laws\&docid=f:publ085. 110.pdf, Last Accessed: January 19, 2008.
[10] "Insuring America's Health: Principles and Recommendations", Committee on Insuring Health", Institute of Medicine, Washington, DC, 2004.

[11] "HealthCast 2020: Creating a Sustainable Future: Executive Summary", A PricewaterhouseCoopers Study, 2005, http://pwchealth.com/cgilocal/hregister.cgi?link=reg/hc2020en.pdf, Last Accessed: January 19, 2008.

[12] "Health-Related Quality of Life", http://www.cdc.gov/hrqol/, Last Accessed: January 20, 2008.

[13] "Crossing the Quality Chasm: A New Health System for the $21^{\text {st }}$ Century", Committee on Quality of Healthcare in America, Institute of Medicine, Washington, DC, 2001.

[14] Park, S., and Jayaraman, S., "Sensor Networks and the $i$-Textiles Paradigm," Proceedings of the Next Generation PC 2005 International Conference, Seoul, Korea, November 3-4, 2005, pp. 163-167.

[15] Gopalsamy, C., Park, S., Rajamanickam, R., and Jayaraman, S., "The Wearable Motherboard ${ }^{\mathrm{TM}}$ : The First Generation of Adaptive and Responsive Textile Structures (ARTS) for Medical Applications", Journal of Virtual Reality, 1999, 4:152-168.

[16] Rajamanickam, R., Park, S., and Jayaraman, S., A Structured Methodology for the Design and Development of Textile Structures in a Concurrent Engineering Environment, Journal of the Textile Institute, 1998; 89, 3: 44-62.

[17] Park, S., and Jayaraman, S., "Enhancing the Quality of Life through Technology: The Role of Personalized Wearable Intelligent Information Infrastructure," IEEE Engineering in Medicine and Biology, May/June 2003, pp. 41-48.

[18] Lukowicz, P., Anliker, U., Ward, J., Tröster, G., Hirt, E., and Neufelt, C., "AMON: A wearable medical computer for high risk patients," in Proc. ISWC 2002, 6th Int. Symp. Wearable Computers, 2002, pp. 133-134.

[19] Lymberis, A., and DeRossi, D., Wearable eHealth Systems for Personalised Health Management, IOS Press, Amsterdam, The Netherlands, August 2004.

[20] DeRossi, D., and Lymberis, A., "New Generation of Smart Wearable Health Systems and Applications," Special Issue of IEEE Transactions on Information Technology in Biomedicine, Vol. 9, No. 3, September 2005.

[21] Nakada, Z., Jones, M., Martin, T., Shenoy, R., "Using electronic textiles to implement an acoustic beamforming array: A case study", Pervasive and Mobile Computing, 3 (2007), 581-606.

[22] Van Halteren, A., Bults, R., Wac, K., Dokovsky, N., Koprinkov, G., Widya, I., Konstantas, D., and Jones, V., "Wireless body area networks for healthcare: The MobiHealth project," Stud. Health Technol. Inform., vol. 108, pp. 181-193, 2004.

[23] Di Rienzo, M., Rizzo, F., Parati, G., Brambilla, G., Ferratini, M., and Castiglioni, P., "MagIC system: A new textile-based wearable device for biological signal monitoring. Applicability in daily life and clinical setting," in Proc. $27^{\text {th }}$ Ann. Int. Conf. IEEE EMBS, Shanghai, September, 2005, pp. 7167-7169.

[24] Lymberis, A., and Dittmar, A., "Advanced Wearable Health Systems and Applications: Research and Development Efforts in the European Union", IEEE Engineering in Medicine and Biology, May/June 2007, pp. 29-33.

[25] Park, S., and Jayaraman, S., "Smart Textiles: Wearable Electronic Systems", MRS Bulletin, August 2003, pp.586-591.

[26] Park, S., and Jayaraman, S., "Wearable Biomedical Systems: Research to Reality", Proceedings of the IEEE Portable 2007 International Conference on Portable Information Devices, Orlando, March 25-27, 2007. 